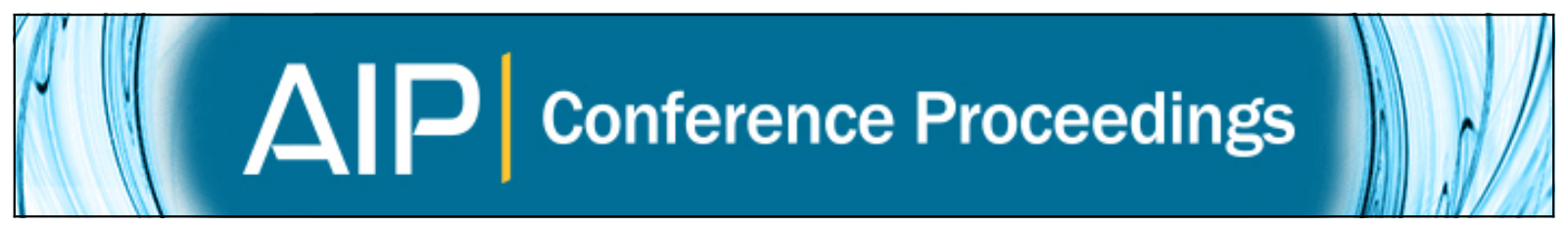

\title{
Project 8: Precision electron specroscopy to measure the mass of the neutrino
}

B. A. VanDevender, D. M. Asner, M. Bahr, R. F. Bradley, S. Doelman, A. M. Jones, J. Fernandes, J. A.

Formaggio, D. Furse, J. F. Kelly, J. Kofron, B. H. LaRoque, M. L. Leber, L. McBride, B. Monreal, N. Oblath, R.

Patterson, A. Rogers, R. G. H. Robertson, L. Rosenberg, G. Rybka, and T. Thümmler

Citation: AIP Conference Proceedings 1560, 237 (2013); doi: 10.1063/1.4826762

View online: http://dx.doi.org/10.1063/1.4826762

View Table of Contents: http://scitation.aip.org/content/aip/proceeding/aipcp/1560?ver=pdfcov

Published by the AIP Publishing

Articles you may be interested in

Absolute neutrino mass measurements

AIP Conf. Proc. 1382, 49 (2011); 10.1063/1.3644268

The Angra Neutrino Project: precise measurement of $\theta 13$ and safeguards applications of neutrino detectors AIP Conf. Proc. 1116, 251 (2009); 10.1063/1.3131564

Precision Solar Neutrino Measurements with the Sudbury Neutrino Observatory

AIP Conf. Proc. 947, 249 (2007); 10.1063/1.2813811

Review of Neutrino Mass Measurements

AIP Conf. Proc. 815, 9 (2006); 10.1063/1.2173571

Direct measurements of neutrino mass

AIP Conf. Proc. 605, 429 (2002); 10.1063/1.1457679 


\title{
Project 8: Precision Electron Specroscopy to Measure the Mass of the Neutrino
}

\author{
B. A. VanDevender*, D. M. Asner*, M. Bahr ${ }^{\dagger}$, R. F. Bradley ${ }^{* *}$, S. \\ Doelman $^{\ddagger}$, A. M. Jones*, J. Fernandes*, J. A. Formaggio ${ }^{\S}$, D. Furse ${ }^{\S}$, J. F. \\ Kelly*, J. Kofron ${ }^{\mathbb{I}}$, B. H. LaRoque ${ }^{\dagger}$, M. L. Leber ${ }^{\dagger}$, L. McBride ${ }^{\mathbb{I}}$, B.

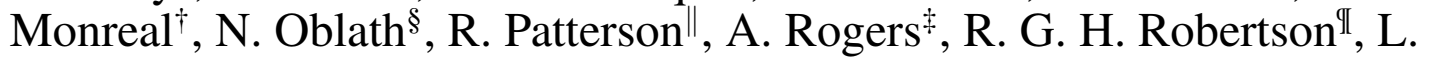 \\ Rosenberg $^{\text {II }}$ G. Rybkal ${ }^{\text {II }}$ and T. Thümmler ${ }^{\dagger \dagger}$ \\ *Pacific Northwest National Lab, Richland, WA \\ ${ }^{\dagger}$ University of California, Santa Barbara, CA \\ ${ }^{* *}$ National Radio Astronomy Observatory, Charlottesville, VA \\ ${ }^{\ddagger}$ Massachusetts Institute of Technology, Haystack Observatory, Westford, MA \\ ${ }^{\S}$ Massachusetts Institute of Technology, Cambridge, MA \\ "Iniversity of Washington, Center for Experimental Nuclear Physics and Astrophysics, Seattle, WA \\ "California Institute of Technology, Pasadena, CA \\ ${ }^{\dagger}$ Karlsruhe Institute of Technology, Karlsruhe, Germany
}

\begin{abstract}
The Project 8 Collaboration is exploring a new technique for the spectroscopy of medium-energy electrons $(\sim 1-100 \mathrm{keV})$ with the ultimate goal of measuring the effective mass of the electron antineutrino by the tritium endpoint method. Our method is based on the detection of microwave-frequency cyclotron radiation emitted by magnetically trapped electrons. The immediate goal of Project 8 is to demonstrate the utility of this technique for a tritium endpoint experiment through a high-precision measurement of the conversion electron spectrum of ${ }^{83 \mathrm{~m}} \mathrm{Kr}$. We present concepts for detecting this cyclotron radiation, focusing on a guided wave design currently being implemented in a prototype apparatus at the University of Washington.
\end{abstract}

Keywords: neutrino, tritium, beta decay, krypton-83, conversion electron, electron spectroscopy, cyclotron radiation, waveguide

PACS: $29.30 . \mathrm{Dm}, 14.60 . \mathrm{Pq}, 23.40 . \mathrm{Bw}, 23.20 . \mathrm{Nx}, 84.40 . \mathrm{Az}$

\section{INTRODUCTION - NEUTRINO MASS SCALE AND HIERARCHY}

Observations of neutrino flavor oscillations indicate that the neutrino flavor eiginstates $v_{l}(l=e, \mu, \tau)$ are an admixture of nondegenerate mass eigenstates $v_{i}(i=1,2,3)$ :

$$
v_{l}=\sum_{i=1}^{3} U_{l i} v_{i}, m_{v l}^{2}=\sum_{i=1}^{3} U_{l i} m_{i}^{2}
$$

where $U$ is the unitary $3 \times 3$ mixing matrix with elements determined by the observed oscillation amplitudes, the $m_{i} \mathrm{~s}$ are mass eigenvalues and the $m_{v l} \mathrm{~s}$ are the effective masses of the flavor eigenstates. The oscillation amplitudes depend only on the differences of the squared mass eigenvalues $\left(\Delta m_{i j}^{2} \equiv m_{i}^{2}-m_{j}^{2}\right)$, so the absolute scale of the masses is not determined. Two independent values have been measured: $\Delta m_{21}^{2}=7.50_{-0.20}^{+0.19} \times 10^{-5} \mathrm{eV}^{2}$ and $\left|\Delta m_{32}^{2}\right|=2.32_{-0.08}^{+0.12} \times 10^{-3} \mathrm{eV}^{2}$ [1]. The sign of the latter term is undetermined, 

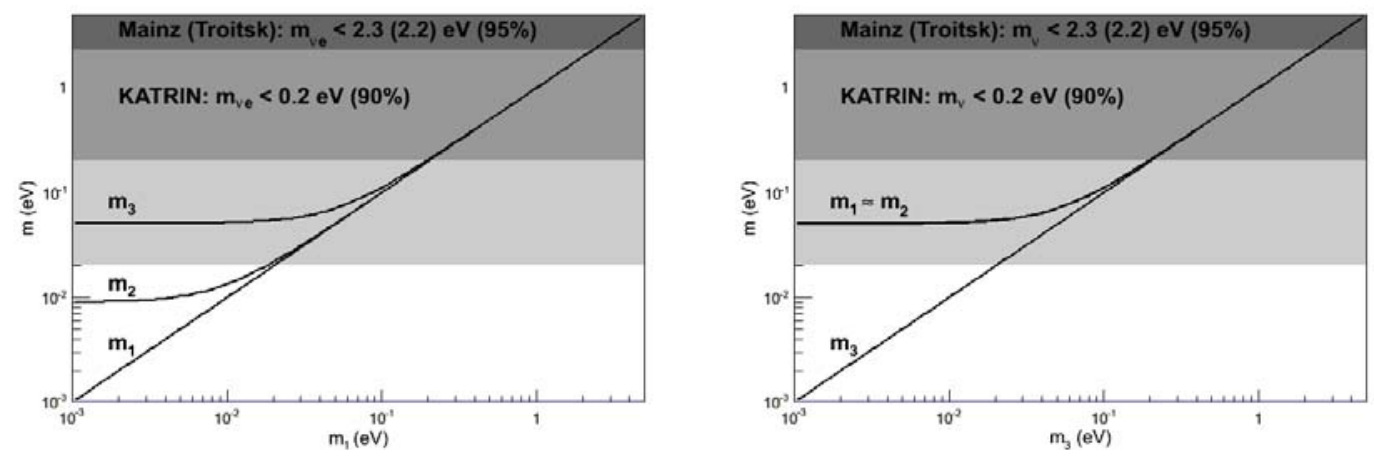

FIGURE 1. The neutrino mass eigenvalues as a function of the lightest eigenvalue in the normal (left) and inverted (right) hierarchies. The shaded regions show the effective electron antineutrino masses excluded by Mainz and Troitsk[2, 3], the exclusion region expected from KATRIN[4] and a speculative exclusion region for a future experiment, showing that one order of magnitude sensitivity improvement beyond KATRIN could resolve the hierarchies. The electron antineutrino has negligible admixture of the $m_{3}$ state, so such an experiment would give a null result for the normal hierarchy, while the inverted hierarchy would result in an observation since $m_{v e} \approx m_{1} \approx m_{2}$ is within experimental sensitivity.

leading to ambiguity in the ordering of the mass eigenvalues. Figure 1 illustrates the possibilities for both the undetermined absolute scale and hierarchy. The determination of these two unknowns is a top priority in experimental particle physics and the goal of the Project 8 experiment.

\section{THE TRITIUM ENDPOINT METHOD WITH PROJECT 8}

The effective mass of the electron antineutrino (hereafter referred to simply as "the neutrino" for brevity) can be determined as a free parameter in a fit to the endpoint region of a tritium beta decay electron spectrum [5]. When tritium decays in a region of uniform $1 \mathrm{~T}$ magnetic field $B$, the daughter electron travels along the field lines in a spiraling cyclotron motion, radiating $\sim 1 \mathrm{fW}$ of power at the cyclotron frequency [6]. The frequency depends on the kinetic energy $E_{e}$ of the electron via the Lorentz factor $\gamma$ :

$$
f=\frac{f_{0}}{\gamma}=\frac{1}{2 \pi} \frac{e B}{m_{e}+E_{e}}
$$

so a measurement of the frequency spectrum is tantamount to a measurement of the energy spectrum, with a $50 \mathrm{kHz}$ band corresponding to $1 \mathrm{eV}$ resolution. An $18.6 \mathrm{keV}$ endpoint electron has $f=26 \mathrm{GHz}$. It takes at least $30 \mu$ s to make such a precise frequency determination, which implies a total electron path length over $2 \mathrm{~km}$.

The Project 8 collaboration is finalizing a prototype apparatus at the University of Washington (Figure 2) that will demonstrate the efficacy of this technique by measuring the conversion electron spectrum of ${ }^{83 \mathrm{~m}} \mathrm{Kr}$, which has monoenergetic lines at 17.8 and $30.2 \mathrm{keV}$. Gaseous ${ }^{83 \mathrm{~m}} \mathrm{Kr}$ at pressure $<10^{-6}$ torr diffuses into the active region inside of a standard WR42 rectangular waveguide region. The region has a uniform $1 \mathrm{~T}$ field provided by a superconducting solenoid plus an additional copper trap coil that provides 


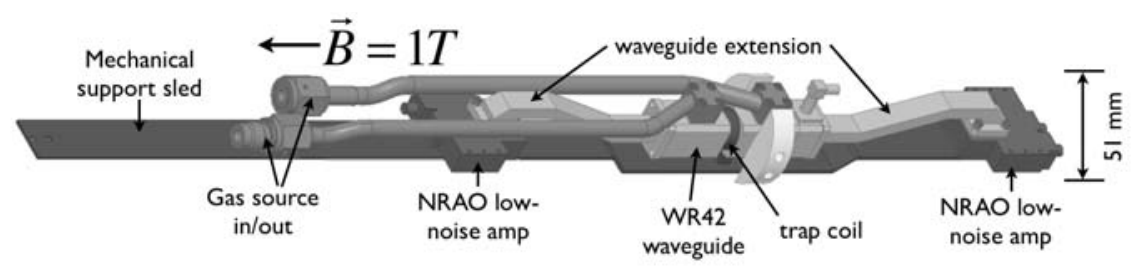

FIGURE 2. The Project 8 waveguide prototype. This assembly is inserted into the two-inch diameter cold bore of a superconducting solenoid. The trap region is centered in the WR42 waveguide, whose extentions transport cyclotron radiation in the $\mathrm{TE}_{10}$ mode to low-noise amplifiers. Source gas diffuses through the active trap region from a high-vacuum system outside the bore.

a shallow magnetic bottle to confine electrons with pitch angles nearly perpendicular to the field to a region centered in the waveguide. Approximately half of the cyclotron radiation power from a trapped electron couples to the $\mathrm{TE}_{10}$ waveguide mode with half of that propagating in each direction towards a NRAO-designed cryogenic low-noise amplifier. These amplifiers condition the raw $26 \mathrm{GHz}$ signals for the room-temperature receiver chain, which mixes them down in two stages to approximately $100 \mathrm{MHz}$ after which they are digitized continuously at $500 \mathrm{MHz}$ sample rate for offline event discovery and analysis. An expected signal has three primary features: a central carrier frequency at approximately $26 \mathrm{GHz}$ as described above, a steady chirp, or frequency rise, of 10$100 \mathrm{kHz}$ due to kinetic energy carried away by the cyclotron radiation, and a warble caused by repetitive rises in frequency during reflections at the ends of the magnetic bottle region and doppler effects.

The Project 8 waveguide prototype is expected to run in late Summer 2012. Detailed calculations of the expected signals and simulation and analysis of the entire chain from waveguide propagation to digitization indicate that we will have sensitivity to any electrons trapped for $\sim 100 \mu$ s. Results from this and future runs will equip the collaboration with the information needed to compute the neutrino mass sensitivity of the method and scale the technique up for a full-scale neutrino mass measurement.

\section{ACKNOWLEDGMENTS}

Project 8 research is supported by the NSF, DOE Office of Nuclear Physics, an MIT Wade Award and the Laboratory Directed Research and Development Program at Pacific Northwest National Laboratory, a multiprogram national laboratory operated by Battelle for the U.S. Department of Energy under Contract DE-AC05-76RL01830

\section{REFERENCES}

1. C. Amsler, et al., Physics Letters B667 (2008).

2. C. Kraus, et al., Eur. Phys. J. C40, 447-468 (2005).

3. V. N. Aseev, et al., Phys. Rev. D 84, 112003 (2011).

4. J. Angrik, et al., KATRIN design report 2004, Tech. rep. (2004), fZKA-7090.

5. E. W. Otten, and C. Weinheimer, Rept. Prog. Phys. 71, 086201 (2008).

6. B. Monreal, and J. A. Formaggio, Phys. Rev. D80, 051301 (2009). 